Eric J. Chow, K. Scott Baker, Stephanie J. Lee, Mary E.D. Flowers, Kara L. Cushing-Haugen, Yoshihiro Inamoto, Wendy M. Leisenring, Karen L. Syrjala, and Paul J. Martin, Fred Hutchinson Cancer Research Center; Eric J. Chow, K. Scott Baker, Seattle Children's Hospital; Eric J. Chow, K. Scott Baker, Stephanie J. Lee, Mary E.D. Flowers, and Paul J. Martin, University of Washington, Seattle, WA; and Nandita Khera, Mayo Clinic, Phoenix, AZ

Published online ahead of print at www.jco.org on December 2, 2013.

Supported by National Institutes of Health Grants No. CA15704, CA18029, CA118953, CA151775, CA160684, and HL36444 and by a Leukemia and Lymphoma Society Special Fellowship in Clinical Research (E.J.C.)

Presented in part at the BMT Tandem Meetings, San Diego, CA, February 1-5, 2012

The content is solely the responsibility of the authors and does not necessarily represent the official views of the

National Institutes of Health.

Authors' disclosures of potential conflicts of interest and author contributions are found at the end of this article.

Corresponding author: Eric J. Chow, $\mathrm{MD}, \mathrm{MPH}$, Fred Hutchinson Cancer Research Center, PO Box 19024, Mailstop M4-C308, Seattle, WA 98109; e-mail: ericchow@u.washington.edu.

C 2013 by American Society of Clinical Oncology

0732-183X/14/3203w-191w/\$20.00 DOI: 10.1200/JCO.2013.52.6582

\title{
Influence of Conventional Cardiovascular Risk Factors and Lifestyle Characteristics on Cardiovascular Disease After Hematopoietic Cell Transplantation
}

Eric J. Chow, K. Scott Baker, Stephanie J. Lee, Mary E.D. Flowers, Kara L. Cushing-Haugen, Yoshihiro Inamoto, Nandita Khera, Wendy M. Leisenring, Karen L. Syrjala, and Paul J. Martin

$$
\begin{array}{llllllll}
\text { A } & \text { B } & \text { S } & \text { T } & \text { R } & \text { A } & \text { C } & \text { T }
\end{array}
$$

\section{Purpose}

To determine the influence of modifiable lifestyle factors on the risk of cardiovascular disease after hematopoietic cell transplantation (HCT).

\section{Patients and Methods}

HCT survivors of $\geq 1$ year treated from 1970 to 2010 ( $n=3,833$ ) were surveyed from 2010 to 2011 on current cardiovascular health and related lifestyle factors (smoking, diet, recreational physical activity). Responses ( $n=2,362$ ) were compared with those from a matched general population sample (National Health and Nutrition Examination Survey [NHANES]; $n=1,192$ ).

\section{Results}

Compared with NHANES participants, HCT survivors (median age, 55.9 years; median 10.8 years since HCT; $71.3 \%$ allogeneic) had higher rates of cardiomyopathy $(4.0 \% \vee 2.6 \%)$, stroke $(4.8 \% \mathrm{~V}$ $3.3 \%$ ), dyslipidemia (33.9\% $\vee 22.3 \%$ ), and diabetes (14.3\% $\vee 11.7 \% ; P<.05$ for all comparisons). Prevalence of hypertension was similar (27.9\% $\vee 30.0 \%$ ), and survivors were less likely to have ischemic heart disease $(6.1 \% \vee 8.9 \% ; P<.01)$. Among HCT survivors, hypertension, dyslipidemia, and diabetes were independent risk factors for ischemic heart disease and cardiomyopathy, and smoking was associated with ischemic heart disease and diabetes (odds ratios [ORs], 1.8 to $2.1 ; P=.02$ ). Obesity was a risk factor for post-transplantation hypertension, dyslipidemia, and diabetes (ORs $\geq 2.0 ; P<.001$ ). In contrast, lower fruit/vegetable intake was associated with greater risk of dyslipidemia and diabetes (ORs, 1.4 to $1.8 ; P \leq .01$ ), and lower physical activity level was associated with greater risk of hypertension and diabetes (ORs, 1.4 to 1.5; $P<$.05). Healthier lifestyle characteristics among HCT survivors attenuated risk of all cardiovascular conditions assessed.

\section{Conclusion}

Attention of clinicians to conventional cardiovascular risk factors and modifiable lifestyle characteristics offers hope of reducing serious cardiovascular morbidity after HCT.

\section{J Clin Oncol 32:191-198. (C) 2013 by American Society of Clinical Oncology}

\section{INTRODUCTION}

More than 60,000 patients are estimated to undergo allogeneic or autologous hematopoietic cell transplantation (HCT) annually worldwide, with an increasing proportion becoming long-term survivors. ${ }^{1}$ Although chronic graft-versus-host disease (GVHD) and recurrent malignancy remain the leading causes of mortality in HCT survivors, survivors also have significantly increased risk of cardiovascular disease compared with the general population. ${ }^{2-7}$ Although some studies have examined the contribution of pretransplantation chemotherapy or radiotherapy and transplantation-related exposures to subsequent cardiovascular health, the influence of post-transplantation lifestyle factors in relation to cardiovascular health in this population has been minimally considered. ${ }^{5}$

The goals of this study were to investigate the distribution of potentially modifiable lifestyle factors (smoking, diet, physical activity) among HCT survivors of $\geq 1$ year compared with the general population and to then determine their associations with serious cardiovascular outcomes (ischemic heart disease, cardiomyopathy/congestive heart failure, stroke) and related conditions (hypertension, dyslipidemia, diabetes, obesity). These lifestyle factors have proven roles in reducing cardiovascular disease in the general population, ${ }^{8}$ but it remains unclear whether they can overcome treatment-related 


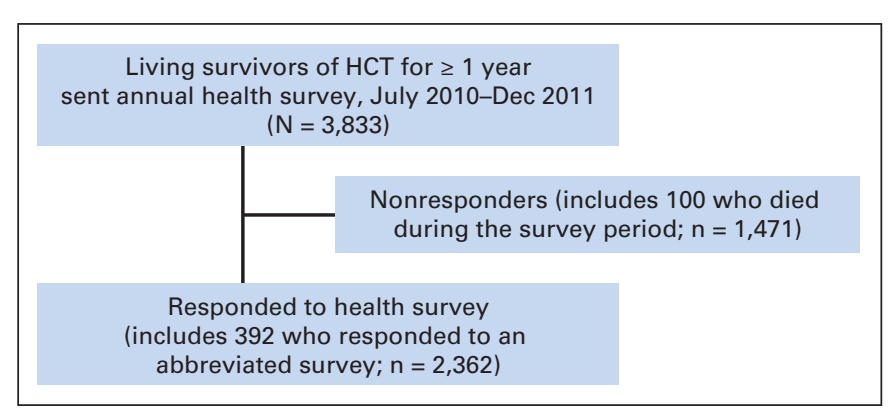

Fig 1. CONSORT diagram. HCT, hematopoietic cell transplantation.

cardiovascular risk among HCT survivors. Results that identify an ongoing role for lifestyle factors could then inform future interventions designed to reduce the burden of cardiovascular disease among HCT and other cancer survivor populations exposed to intense multimodal therapies.

\section{PATIENTS AND METHODS}

\section{HCT Survivors}

All patients who undergo HCT at the Fred Hutchinson Cancer Research Center (FHCRC) are mailed annually a comprehensive health status survey. Between July 2010 and September 2011, a supplemental questionnaire focusing on cardiovascular health was included with the annual survey. Of the 3,833 patients currently age $>19$ years who underwent transplantation from 1970 to 2010 who were mailed the survey, 1,970 responded after two mailings. A third mailing was sent to remaining individuals, using an abbreviated questionnaire, with 392 responses as of February 2012, resulting in an overall response rate of $61.6 \%$ (2,362 of 3,833; Fig 1). Detailed demographic information, HCTrelated exposures (eg, donor type, stem-cell source, conditioning regimen), and post-transplantation events (eg, chronic GVHD, relapse, death) were available from the FHCRC research database. To assess potential response bias leading to differential rates of cardiovascular disease among responders versus nonresponders, a subset of patients $(833$ of 3,833$)$ also had linked state administrative hospitalization discharge data available. ${ }^{6}$ Patients consented to the use of their questionnaire and medical record data, with approval from the FHCRC Institutional Review Board. The Washington state institutional review board approved our use of state hospitalization data.

\section{Survey Instrument and Definitions}

Outcomes (yes/no, age of first/last occurrence) of primary interest were ischemic heart disease, cardiomyopathy/congestive heart failure, and stroke and of secondary interest were hypertension, dyslipidemia, and diabetes. We restricted our definition of these three secondary outcomes to respondents who required treatment for these conditions and, among HCT survivors, to those receiving at least 30 days of treatment within the past year. Self-reported height and weight were converted to body-mass index (BMI) and categorized as underweight, normal, overweight, and obese ( $<18.5,18.5$ to 24,25 to 29 , and $\geq 30 \mathrm{~kg} / \mathrm{m}^{2}$, respectively). Smoking was defined as never, before HCT but not currently, after HCT but not currently, and current smoker. Additional questions assessed health care use (eg, frequency of seeking primary and/or oncology care) and family history (first-degree relatives) of cardiovascular and related diseases. Average daily fruit/vegetable intake over the past month was ascertained, and respondents were categorized as meeting or not meeting national recommendations for $\geq$ five daily servings of fruits and vegetables. ${ }^{9}$ Respondents also estimated their work-related and recreational physical activity levels (both current and before the illness that led to transplantation). For recreational physical activity, the self-reported amount of time spent doing vigorous and moderate activities over the past week was summed with every 2 minutes of moderate activity equivalent to 1 minute of vigorous activity. ${ }^{10}$ Respondents were categorized as meeting or not meeting national recommen- dations for aerobic activities: 75 minutes per week of vigorous activity, 150 minutes per week of moderate activity, or equivalent mixture of both. ${ }^{10}$ Physical activity categorization based on respondents reporting no moderate or vigorous activities (referent group) and remaining respondents categorized using activity time tertiles was also examined. To facilitate comparisons with external populations, whenever possible, questions were taken or adapted from other well-established surveys, such as the National Health and Nutrition Examination Survey (NHANES) ${ }^{11}$ and the Childhood Cancer Survivor Study. ${ }^{12}$ The abbreviated questionnaire did not assess diet or physical activity. Copies of the questionnaires are available on request.

\section{Comparison Group}

Data from the 2009 to 2010 NHANES were used to establish a general population comparison group. ${ }^{11}$ This was the most recent NHANES data set fully available at the time. To permit direct comparisons with HCT survivors, the NHANES data set was frequency matched to survivors by sex, 10-year age intervals, and race/ethnicity. Approximately one NHANES participant for every two HCT survivors met our matching criteria and formed the comparison group $(n=1,192)$. Our NHANES sample included those who reported a prior history of cancer $(\mathrm{n}=160 ; 13.4 \%)$. Geographic variables were not available in the publically accessible NHANES data sets.

\section{Statistical Analysis}

We calculated the prevalence of outcomes among the HCT and NHANES cohorts. Differences between the two groups were estimated using both univariate methods and multivariable logistic regression adjusted for sex, race/ethnicity, current age, and BMI category, with estimates expressed as odds ratios (ORs) with 95\% CIs. Additional multivariable models examined the influence of lifestyle factors (smoking, fruit/vegetable consumption, recreational physical activity) on comparisons between the two cohorts. We then evaluated risk factors within the HCT cohort only, excluding individuals who reported developing the outcome of interest before transplantation. This analysis was adjusted for sex, race/ethnicity, current age, age at and time since transplantation, HCT-related exposures (donor source, any clinical chronic GVHD), subsequent relapse or need for additional transplantation, family history of cardiovascular disease, and routine physical examination within the past year. Analyses of ischemic heart disease, cardiomyopathy, and stroke after HCT also accounted for BMI category and use of medication to treat hypertension, dyslipidemia, or diabetes.

We then examined the influence of lifestyle factors on both the primary and secondary outcomes of interest. Because lifestyle factors may mediate some of their effects via development of hypertension, dyslipidemia, diabetes, and obesity, we analyzed models with and without adjustment for these conditions. Finally, to evaluate the potential proportion of cardiovascular disease that might be reduced by eliminating certain risk factors, we estimated the attributable risk percent $(\mathrm{AR} \%)$ for these factors in relation to our study outcomes among exposed respondents. This assumes that the risk factor has a causal relationship with these outcomes. Analyses were performed using STATA software (version 12; STATA, College Station, TX).

\section{RESULTS}

Compared with nonresponders, HCT survivors who participated were more likely to be female $(46.7 \%$ v $41.1 \%)$, white non-Hispanic $(89.2 \% v 82.7 \%)$, and older currently (median age, $55.9 v 49.5$ years) and at time of transplantation (median age, $44.0 v 35.7$ years) and to have a history of chronic GVHD (63.1\% v 56.9\%; Table 1). Responders and nonresponders were similar with regard to Washington state residency, underlying diagnosis, donor type, and post-HCT relapse or need for additional transplantation. We found no statistically significant differences in the prevalence of cardiovascular and related outcomes among a subset of responders and nonresponders with available state hospitalization discharge data (Appendix Table A1, online only). Median time since HCT among respondents was 10.8 


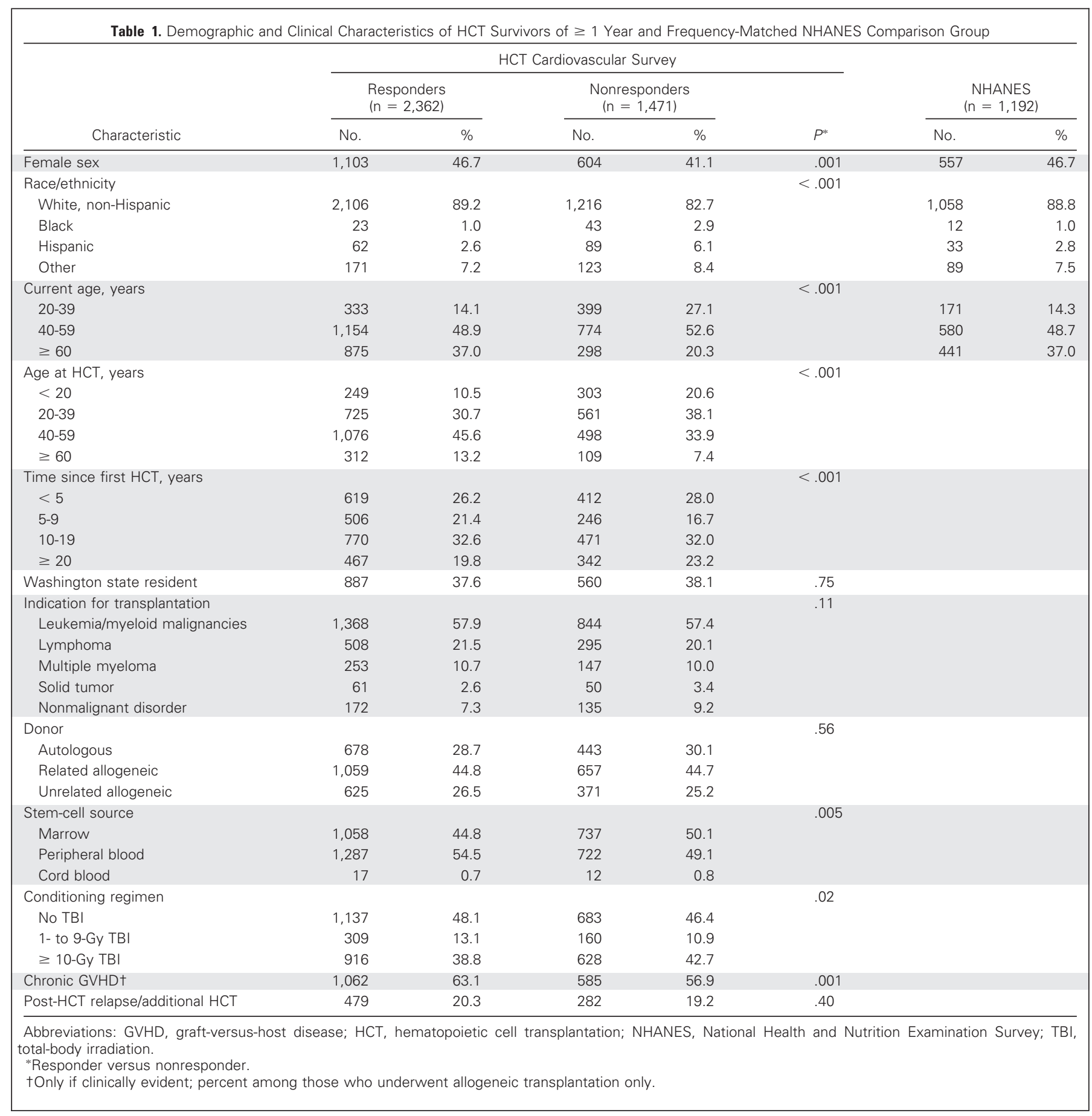

years (range, 0.9 to 40.4 years), and $72.4 \%$ of respondents were from out of state.

Compared with NHANES participants, HCT survivors had lower prevalence of ischemic heart disease and obesity but were more likely to report cardiomyopathy, stroke, dyslipidemia, and diabetes (Table 2). Among survivors, prevalence of ischemic heart disease increased from $6.1 \%$ to $6.9 \%(n=163)$ if surgical information (coronary artery bypass, angioplasty) not specifically assessed by NHANES was included. Prevalence of hypertension was similar in the two groups. However, the HCT cohort reported healthier lifestyles, with lower rates of smoking and greater daily fruit/vegetable intake and recreational physical activity levels $(P<.01$ for all comparisons). More HCT survivors reported either not working or work activity characterized as sedentary $(75.2 \% v 57.8 \%$ in NHANES; $P<.001)$.

Comparisons of the NHANES and HCT cohorts after adjusting for baseline demographic characteristics plus BMI showed significantly lower risk of ischemic heart disease and significantly higher risk of stroke, dyslipidemia, and diabetes associated with HCT (model one; Fig 2; Appendix Table A2, online only). When differences in lifestyle factors across the two cohorts were accounted for (model two), the 


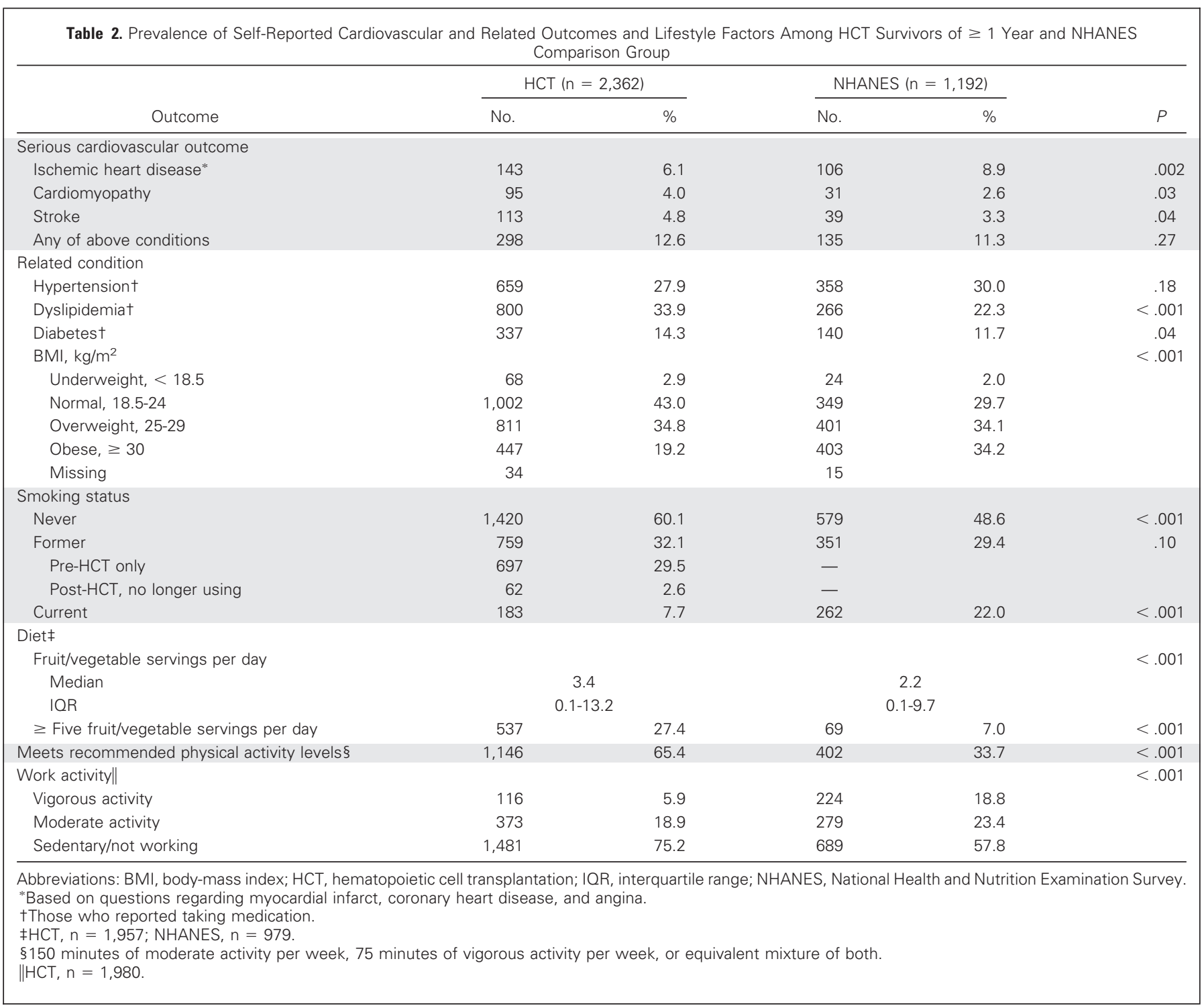

relative risks associated with HCT increased for all outcomes, including ischemic heart disease, suggesting that better lifestyle characteristics may attenuate any underlying risk associated with prior HCT. When model two was analyzed separately by autologous or allogeneic donor status (Appendix Table A2, online only), the magnitude of risk for autologous survivors generally was lower than that for allogeneic survivors when compared with NHANES participants, except for greater risk of cardiomyopathy (OR, 3.3; 95\% CI, 1.6 to 6.8). Model two, stratified by chronic GVHD history, showed that risk for hypertension, dyslipidemia, and diabetes remained increased among allogeneic survivors regardless of GVHD history when compared with NHANES participants (ORs $>2 ; P<.001$ ).

In analyses restricted to HCT survivors, history of chronic GVHD seemed to be associated with increased risk of most outcomes other than cardiomyopathy (Table 3 ). When we examined only $\geq 5$ year survivors (excluding most patients who may still have active chronic GVHD), most risk estimates remained similar, except that association with dyslipidemia was attenuated (OR, 1.2; $P=.30$ ), suggesting that other associations with GVHD were unlikely to be completely explained by adverse effects of concurrent immunosuppressive therapy. Overall, survivors with ischemic heart disease or cardiomyopathy were more likely to be taking blood pressure, lipid, or diabetes medications compared with those without these conditions. Obesity was much more common among survivors with hypertension, dyslipidemia, or diabetes $(\mathrm{ORs} \geq 2.0 ; P<.001)$ but not among those who had already developed more serious cardiovascular outcomes. In general, associations for ischemic heart disease, cardiomyopathy, and stroke were significantly increased with greater numbers of adverse related conditions (obesity, hypertension, dyslipidemia, diabetes; trend test $P<.001$ for all outcomes). This association was strongest for ischemic heart disease ( $\geq$ three conditions; OR, 10.4; 95\% CI, 5.4 to 20.0 ).

When influence of lifestyle factors on cardiovascular outcomes was examined, current smoking was associated with ischemic heart disease and diabetes compared with never-smokers (ORs, 1.8 to 2.1; $P=.02$; Table 3 ). These estimates remained significant if post-HCT 


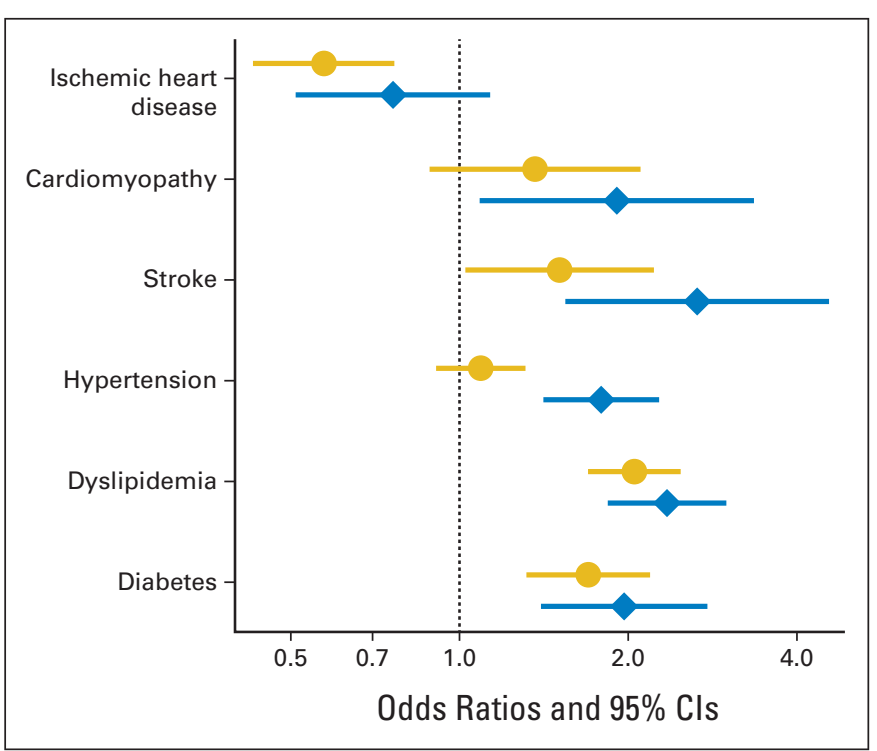

Fig 2. Risk (odds ratios plus $95 \% \mathrm{Cls}$ ) of cardiovascular and related outcomes among hematopoietic cell transplantation survivors of $\geq 1$ year compared with National Health and Nutrition Examination Survey participants (referent) before (model one; circles) and after adjustment for lifestyle factors (model two; diamonds). Model one adjusted for sex, nonwhite race/ethnicity, current age, and body mass index; model two included additional adjustment for current smoking, daily fruit/vegetable intake, and recreational physical activity time.

smokers who had since quit were also grouped with current smokers (data not shown). Lower fruit/vegetable intake was associated with greater risk of all outcomes, particularly dyslipidemia and diabetes ( $<$ five fruit/vegetable servings per day; ORs, 1.4 to 1.8; $P \leq .01)$. Not surprisingly, survivors with serious cardiovascular disease were less likely to be currently physically active (ORs, 1.7 to $3.1 ; P \leq .01)$. However, those with only hypertension or diabetes also were more likely to not meet recommended physical activity levels (ORs, 1.4 to $1.5 ; P<.05$ ). Physical activity level before illness that led to transplantation was not associated with any outcome (data not shown). Trend tests for the additive association of current smoking, low fruit/vegetable intake, and low physical activity levels were statistically significant $(P \leq .02)$ for all outcomes except dyslipidemia. Results were similar when models were also adjusted for concurrent presence of hypertension, dyslipidemia, diabetes, and obesity, except that the association between low fruit/vegetable intake and dyslipidemia was attenuated and was no longer statistically significant (OR, 1.2; 95\% CI, 0.9 to 1.6 ).

In an analysis of potential risk reduction for serious cardiovascular outcomes (ischemic heart disease, cardiomyopathy, stroke) associated with risk factors of interest, controlling dyslipidemia was associated with the greatest reduction (AR\% ranged from $46 \%$ to $77 \%$; Fig $3 \mathrm{~A}$ ), although the estimated reduction associated with controlling hypertension, diabetes, and smoking tended to be approximately $30 \%$ for most serious outcomes. AR\% for obesity in relation to hypertension, dyslipidemia, and diabetes ranged from 34\% to 48\% (Fig 3B). Potential influence of smoking, diet, and physical activity on these three conditions was more modest (AR\% $\leq 25 \%)$. Magnitude of the estimated AR\%s among HCT survivors was variable compared with estimates derived from our matched NHANES group (Appendix Table A3, online only).

\section{DISCUSSION}

Despite reporting healthier lifestyle characteristics than the general population, HCT survivors continued to have a greater burden of most cardiovascular conditions, which is likely the consequence of pretransplantation and transplantation-related therapeutic exposures. One exception was the lower prevalence of ischemic heart disease, which may partially reflect the observation that pre-existing ischemic heart disease has often been considered a relative or even absolute contraindication for HCT. ${ }^{13}$ Prevalence rates also would seem lower if ischemic heart disease were associated with greater mortality among transplant recipients compared with the general population. We have previously shown that those who underwent HCT, observed longitudinally, have a greater cumulative incidence of ischemic heart disease and all-cause cardiovascular mortality compared with the general population. ${ }^{6}$

Importantly, adherence to recommended lifestyle choices was associated with a reduced risk of all cardiovascular outcomes after HCT. Although longitudinal follow-up of our cohort will better demonstrate any causal relationship between these lifestyle factors and subsequent outcomes, our results suggest that improved adherence to lifestyle recommendations by patients and attention to effective control of hypertension, dyslipidemia, and diabetes by clinicians may reduce the risk of cardiovascular morbidity in this heavily treated population predominantly comprised of cancer survivors.

Excluding case reports, few studies have been published focusing on more serious cardiovascular disease among HCT survivors of $\geq 1$ year. ${ }^{3-7,14-17}$ HCT survivors with conventional cardiovascular risk factors such as obesity, hypertension, dyslipidemia, and diabetes seem to have a substantially greater risk of more serious cardiovascular disease, even after accounting for cardiotoxic cancer treatments, similar to findings from general cancer survivors. ${ }^{18,19}$ The burden of these conventional cardiovascular risk factors seemed to be greater after allogeneic versus autologous HCT. ${ }^{3,4,6,7}$ Given the critical role of chronic inflammation in cardiovascular disease pathogenesis, it has been hypothesized that GVHD-associated inflammation may drive the increased risk observed after allogeneic HCT. ${ }^{20,21}$ Although results from previous studies have shown either no association ${ }^{3,4}$ or association with acute rather than chronic GVHD, ${ }^{5,7}$ we found some support for this hypothesis. Both autologous and allogeneic HCT conditioning regimens may also induce acute deleterious effects on vascular endothelium, although what effect this has on long-term cardiovascular health remains unknown. ${ }^{22,23}$

Information regarding the influence of potentially modifiable behavioral factors among HCT survivors has been even more limited. A European multi-institutional study found that among 548 HCT survivors, those who experienced arterial events were less physically active and more likely to have smoked after HCT. ${ }^{5}$ A study of 1,040 HCT survivors from two US centers reported that survivors were less likely to smoke and more likely to engage in some, but not all, cancer screening behaviors compared with siblings. ${ }^{24}$ In other cancer survivor populations, obesity and physical inactivity have been linked to subsequent development of cardiovascular disease, ${ }^{25}$ and adherence to lifestyle recommendations has been associated with a reduction in all-cause and cardiovascular disease-specific mortality. ${ }^{26}$ The unique contributions of our study included its focus on HCT and 
Table 3. Association of Select Characteristics With Cardiovascular and Related Outcomes Among HCT Survivors of $\geq 1$ Year

\begin{tabular}{|c|c|c|c|c|c|c|c|c|c|c|c|c|}
\hline \multirow[b]{2}{*}{ Characteristic } & \multicolumn{2}{|c|}{$\begin{array}{l}\text { Ischemic Heart } \\
\text { Disease } \\
(\mathrm{n}=134)^{*} \\
\end{array}$} & \multicolumn{2}{|c|}{$\begin{array}{l}\text { Cardiomyopathy } \\
(\mathrm{n}=85)^{*}\end{array}$} & \multicolumn{2}{|c|}{ Stroke $(\mathrm{n}=99)^{*}$} & \multicolumn{2}{|c|}{$\begin{array}{c}\text { Hypertension } \\
(\mathrm{n}=543)^{*} \dagger\end{array}$} & \multicolumn{2}{|c|}{$\begin{array}{l}\text { Dyslipidemia } \\
(\mathrm{n}=717)^{*}+\end{array}$} & \multicolumn{2}{|c|}{$\begin{array}{c}\text { Diabetes } \\
(n=309)^{*}+\end{array}$} \\
\hline & OR & $95 \% \mathrm{Cl}$ & OR & $95 \% \mathrm{Cl}$ & OR & $95 \% \mathrm{Cl}$ & OR & $95 \% \mathrm{Cl}$ & OR & $95 \% \mathrm{Cl}$ & OR & $95 \% \mathrm{Cl}$ \\
\hline Among $\geq 5$-year survivors only & 2.3 & 1.3 to 4.0 & 1.1 & 0.5 to 2.1 & 2.0 & 1.1 to 3.6 & 1.3 & 0.96 to 1.8 & 1.2 & 0.9 to 1.6 & 1.7 & 1.1 to 2.5 \\
\hline Hypertension $v$ none & 1.6 & 1.1 to 2.4 & 2.1 & 1.3 to 3.4 & 1.4 & 0.9 to 2.3 & - & & - & & - & \\
\hline Dyslipidemia $v$ none & 3.8 & 2.5 to 5.9 & 2.0 & 1.2 to 3.4 & 1.9 & 1.2 to 3.0 & - & & - & & - & \\
\hline Underweight, $<18.5$ & 0.7 & 0.1 to 5.6 & 3.3 & 1.2 to 9.4 & 0.8 & 0.2 to 3.3 & 1.1 & 0.5 to 2.3 & 0.2 & 0.1 to 0.8 & 0.6 & 0.2 to 2.0 \\
\hline Normal, 18.5-24 & 1.0 & Ref & 1.0 & Ref & 1.0 & Ref & 1.0 & Ref & 1.0 & Ref & 1.0 & Ref \\
\hline Overweight, $25-29$ & 1.1 & 0.7 to 1.6 & 0.8 & 0.5 to 1.4 & 0.9 & 0.6 to 1.5 & 1.3 & 0.97 to 1.7 & 1.9 & 1.5 to 2.5 & 1.4 & 0.99 to 2.0 \\
\hline Obese, $\geq 30$ & 0.9 & 0.5 to 1.5 & 0.6 & 0.3 to 1.2 & 1.3 & 0.8 to 2.3 & 2.2 & 1.6 to 3.0 & 2.0 & 1.5 to 2.7 & 2.5 & 1.8 to 3.7 \\
\hline Additive effect of adverse conditions $\ddagger$ & 2.0 & 1.7 to 2.5 & 1.6 & 1.3 to 2.0 & 1.5 & 1.2 to 1.8 & - & & - & & - & \\
\hline$P$ & & $<.001$ & & $<.001$ & & $<.001$ & & & & & & \\
\hline$P$ & & .007 & & $<.001$ & & $<.001$ & & .02 & & .08 & & .003 \\
\hline
\end{tabular}

NOTE. Estimates for each outcome adjusted for sex, race/ethnicity, current age, age at and time since transplant, donor type, post-transplant relapse, family history

of cardiovascular disease, routine physical exam within the past year, and chronic graft versus host disease history. Bold font indicates significance.

Abbreviations: BMI, body-mass index; GVHD, graft-versus-host disease; HCT, hematopoietic cell transplantation; OR, odds ratio.

*Excluding individuals who reported condition onset before HCT.

†Those who reported taking medication and without history of serious cardiovascular disease (ischemic heart disease, cardiomyopathy, stroke).

FTrend test examining additive effect of hypertension, dyslipidemia, diabetes, and obesity.

§Recommended 150 minutes of moderate activity per week, 75 minutes of vigorous activity per week, or equivalent mixture of both.

$\|$ Trend test examining additive effect of current smoking, $<$ five fruits/vegetables per day, and not meeting recommended physical activity levels.

its composite examination of multiple cardiovascular outcomes and lifestyle factors.

Although our analytic cohort represented $62 \%$ of eligible survivors, we did not observe any systematic evidence of response bias with regard to hospitalization for cardiovascular disease in the subset of survivors with state administrative data. Use of similar or matching survey language between the HCT and NHANES cohorts enabled comparisons with a well-established general population reference. Although the sensitivity and specificity of self-report can vary by medical condition, the inherent validity of our comparisons would only be affected if the two groups had differential misclassification rates and if the magnitude and/or direction of those rates varied by cohort. In general, nondifferential misclassification biases result toward the null.
A

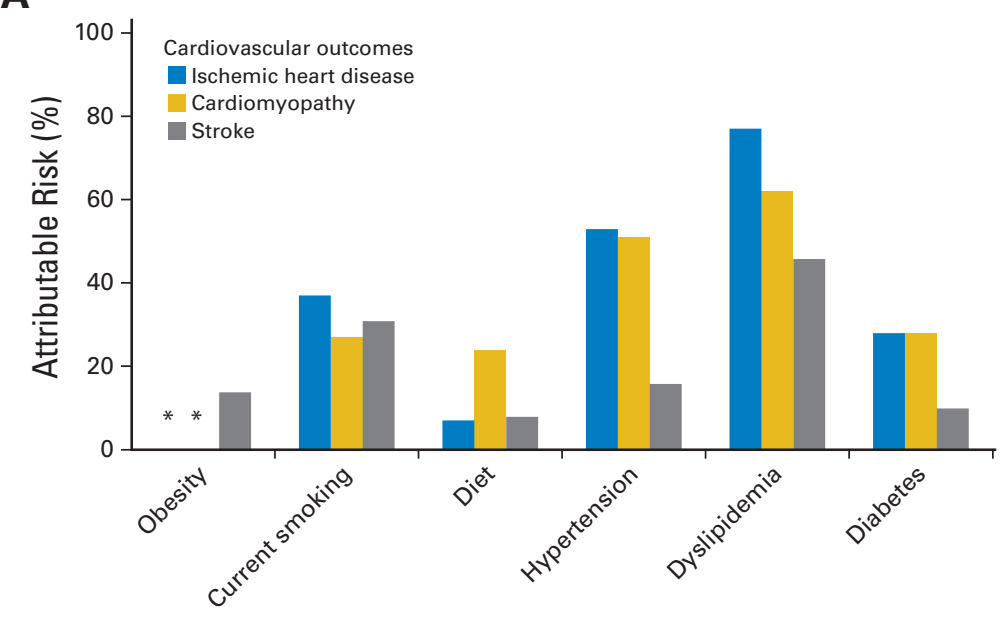

B

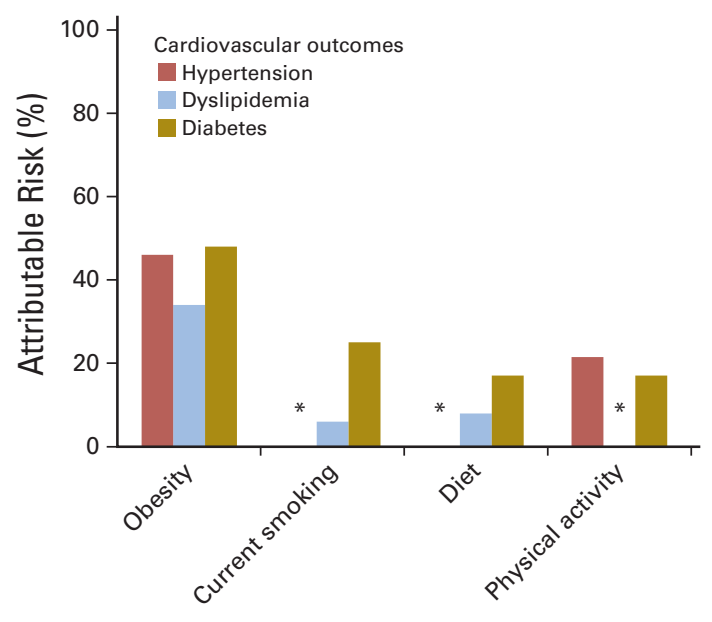

Fig 3. Attributable risk percent (AR\%) associated with risk factors ( $x$-axis) for (A) serious cardiovascular outcomes and (B) related conditions among exposed

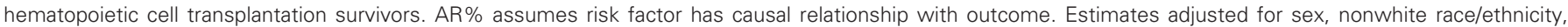
current age, obesity, current smoking, diet, recreational physical activity time, and, if applicable, hypertension, dyslipidemia, and diabetes status. $\left.{ }^{*}\right)$ AR $\%<1 \%$. 
Some regional differences in cardiovascular health exist, both for the general population and among cancer survivors, with southern US states on average having worse cardiovascular health and related lifestyle characteristics compared with other parts of the nation. ${ }^{27,28}$ Our survivor cohort likely has a lower proportion of residents from southern states (17.5\%) compared with our NHANES sample, which may account for some of the observed disparities in obesity and lifestyle between cohorts. However, if our general population sample had a healthier cardiovascular and lifestyle profile, the baseline differences between the HCT cohort and general population would have been even further accentuated.

Our attributable risk estimates provide the best-case percent improvement from complete elimination of each adverse exposure. Although we recognize that complete risk elimination is unrealistic, such estimates allow greater appreciation of the potential impact of introducing certain interventions among at-risk survivors. It also is important to note that although we restricted our definitions of those with hypertension, dyslipidemia, and diabetes in both the HCT and NHANES cohorts to patients taking medications for these conditions, we lacked information to indicate whether these conditions were actually controlled and the extent to which these conditions may have been undiagnosed and therefore untreated. Prevalence of these conditions among HCT survivors may be increased if survivors are screened more frequently than the general population. At the same time, reports have suggested that hypertension and dyslipidemia are often undertreated among HCT survivors. ${ }^{29,30}$ It also remains unclear whether conventional control of these risk factors among survivors exposed to radiotherapy and cardiotoxic chemotherapy has the same efficacy as in the general population.

In summary, our results lend further credence to the importance of addressing conventional cardiovascular risk factors such as obesity, hypertension, dyslipidemia, and diabetes ${ }^{31}$ as well as healthier lifestyle choices among HCT survivors, as recommended by the international consensus HCT long-term follow-up guidelines. ${ }^{32}$ Although our cohort reported healthier lifestyles compared with the general population, much room for improvement remains, particularly in relation to diet and physical activity and smoking reduction. Given that the cancer experience may serve as significant motivation for adopting healthier lifestyles, ${ }^{33}$ clinicians and researchers should take advantage of this teachable moment to introduce interventions that foster and support such lifestyle changes. ${ }^{2,34}$ Such interventions have been tested in cancer survivors, including HCT recipients, with some short- and medium-term improvements observed. ${ }^{35-38}$

\section{AUTHORS' DISCLOSURES OF POTENTIAL CONFLICTS} OF INTEREST

The author(s) indicated no potential conflicts of interest.

\section{AUTHOR CONTRIBUTIONS}

Conception and design: Eric J. Chow, K. Scott Baker, Stephanie J. Lee, Karen L. Syrjala, Paul J. Martin

Financial support: Eric J. Chow, Stephanie J. Lee, Paul J. Martin

Administrative support: Eric J. Chow, Stephanie J. Lee, Paul J. Martin

Provision of study materials or patients: Mary E.D. Flowers, Paul

J. Martin

Collection and assembly of data: Eric J. Chow, Mary E.D. Flowers, Paul J. Martin

Data analysis and interpretation: All authors

Manuscript writing: All authors

Final approval of manuscript: All authors

\section{REFERENCES}

1. Pasquini MC, Wang Z: Current use and outcome of hematopoietic stem cell transplantation: Summary slides 2012. http://www.cibmtr.org/ReferenceCenter/ SlidesReports/SummarySlides/index.html

2. Syrjala KL, Martin PJ, Lee SJ: Delivering care to long-term adult survivors of hematopoietic cell transplantation. J Clin Oncol 30:3746-3751, 2012

3. Baker KS, Ness KK, Steinberger J, et al: Diabetes, hypertension, and cardiovascular events in survivors of hematopoietic cell transplantation: A report from the bone marrow transplantation survivor study. Blood 109:1765-1772, 2007

4. Tichelli $A$, Bucher $C$, Rovó $A$, et al: Premature cardiovascular disease after allogeneic hematopoietic stem-cell transplantation. Blood 110:3463-3471, 2007

5. Tichelli A, Passweg J, Wójcik D, et al: Late cardiovascular events after allogeneic hematopoietic stem cell transplantation: A retrospective multicenter study of the Late Effects Working Party of the European Group for Blood and Marrow Transplantation. Haematologica 93:12031210, 2008

6. Chow EJ, Mueller BA, Baker KS, et al: Cardiovascular hospitalizations and mortality among recipients of hematopoietic stem cell transplantation. Ann Intern Med 155:21-32, 2011

7. Armenian $\mathrm{SH}$, Sun $\mathrm{CL}$, Vase $T$, et al: Cardiovascular risk factors in hematopoietic cell transplan- tation survivors: Role in development of subsequent cardiovascular disease. Blood 120:4505-4512, 2012

8. Heidenreich PA, Trogdon JG, Khavjou OA, et al: Forecasting the future of cardiovascular disease in the United States: A policy statement from the American Heart Association. Circulation 123:933944, 2011

9. Blanck HM, Gillespie C, Kimmons JE, et al: Trends in fruit and vegetable consumption among U.S. men and women, 1994-2005. Prev Chronic Dis 5:A35, 2008

10. Centers for Disease Control and Prevention: Physical activity for everyone. http://www.cdc.gov/ physicalactivity/everyone/guidelines/index.html

11. Centers for Disease Control and Prevention: NHANES: Questionnaires, datasets, and related documentation. http://www.cdc.gov/nchs/nhanes/ nhanes_questionnaires.htm

12. Robison LL, Armstrong GT, Boice JD, et al: The Childhood Cancer Survivor Study: A National Cancer Institute-supported resource for outcome and intervention research. J Clin Oncol 27:23082318, 2009

13. Coghlan JG, Handler CE, Kottaridis PD: Cardiac assessment of patients for haematopoietic stem cell transplantation. Best Pract Res Clin Haematol 20:247-263, 2007

14. Armenian $\mathrm{SH}$, Sun $\mathrm{CL}$, Francisco $L$, et al: Late congestive heart failure after hematopoietic cell transplantation. J Clin Oncol 26:5537-5543, 2008

15. Uderzo C, Pillon M, Tridello G, et al: Cardiac and pulmonary late effects do not negatively influ- ence performance status and non-relapse mortality of children surviving five yr after autologous hematopoietic cell transplantation: Report from the EBMT Paediatric Diseases and Late Effects Working Parties. Pediatr Transplant 13:719-724, 2009

16. Armenian $\mathrm{SH}$, Sun $\mathrm{CL}$, Mills G, et al: Predictors of late cardiovascular complications in survivors of hematopoietic cell transplantation. Biol Blood Marrow Transplant 16:1138-1144, 2010

17. Armenian $S H$, Sun $C L$, Shannon $T$, et al: Incidence and predictors of congestive heart failure after autologous hematopoietic cell transplantation. Blood 118:6023-6029, 2011

18. Darby SC, Ewertz M, McGale P, et al: Risk of ischemic heart disease in women after radiotherapy for breast cancer. N Engl J Med 368:987-998, 2013

19. Armstrong GT, Oeffinger $\mathrm{KC}$, Chen $\mathrm{Y}$, et al: Modifiable risk factors and major cardiac events among adult survivors of childhood cancer. J Clin Oncol 31:3673-3680, 2013

20. Biedermann BC: Vascular endothelium and graft-versus-host disease. Best Pract Res Clin Haematol 21:129-138, 2008

21. Rovó A, Daikeler T, Halter J, et al: Late altered organ function in very long-term survivors after allogeneic hematopoietic stem cell transplantation: A paired comparison with their HLA-identical sibling donor. Haematologica 96:150-155, 2011

22. Woywodt A, Scheer J, Hambach L, et al: Circulating endothelial cells as a marker of endothelial damage in allogeneic hematopoietic stem cell transplantation. Blood 103:3603-3605, 2004 
23. Palomo $M$, Diaz-Ricart $M$, Carbo $C$, et al: Endothelial dysfunction after hematopoietic stem cell transplantation: Role of the conditioning regimen and the type of transplantation. Biol Blood Marrow Transplant 16:985-993, 2010

24. Armenian $S H$, Sun $C L$, Francisco $L$, et al: Health behaviors and cancer screening practices in long-term survivors of hematopoietic cell transplantation (HCT): A report from the BMT Survivor Study. Bone Marrow Transplant 47:283-290, 2012

25. Hawkes AL, Lynch BM, Owen N, et al: Lifestyle factors associated concurrently and prospectively with co-morbid cardiovascular disease in a population-based cohort of colorectal cancer survivors. Eur J Cancer 47:267-276, 2011

26. Inoue-Choi M, Robien K, Lazovich D: Adherence to the WCRF/AICR guidelines for cancer prevention is associated with lower mortality among older female cancer survivors. Cancer Epidemiol Biomarkers Prev 22:792-802, 2013

27. Fang J, Yang $Q$, Hong $Y$, et al: Status of cardiovascular health among adult Americans in the 50 states and the District of Columbia, 2009. J Am Heart Assoc 1:e005371, 2012

28. Underwood JM, Townsend JS, Stewart SL, et al: Surveillance of demographic characteristics and health behaviors among adult cancer survivors: Behavioral Risk Factor Surveillance System, United States, 2009. MMWR Surveill Summ 61:123, 2012

29. Griffith ML, Savani BN, Boord JB: Dyslipidemia after allogeneic hematopoietic stem cell transplantation: Evaluation and management. Blood 116 : 1197-1204, 2010

30. Blaser BW, Kim HT, Alyea EP 3rd, et al: Hyperlipidemia and statin use after allogeneic hematopoietic stem cell transplantation. Biol Blood Marrow Transplant 18:575-583, 2012

31. Rovó A, Tichelli A: Cardiovascular complications in long-term survivors after allogeneic hematopoietic stem cell transplantation. Semin Hematol 49:25-34, 2012

32. Majhail NS, Rizzo JD, Lee SJ, et al: Recommended screening and preventive practices for long-term survivors after hematopoietic cell transplantation. Biol Blood Marrow Transplant 18:348371, 2012

33. Demark-Wahnefried W, Aziz NM, Rowland $\mathrm{JH}$, et al: Riding the crest of the teachable moment: Promoting long-term health after the diagnosis of cancer. J Clin Oncol 23:5814-5830, 2005
34. Weaver KE, Foraker RE, Alfano $\mathrm{CM}$, et al: Cardiovascular risk factors among long-term survivors of breast, prostate, colorectal, and gynecologic cancers: A gap in survivorship care? J Cancer Surviv 7:253-261, 2013

35. Morey MC, Snyder DC, Sloane R, et al: Effects of home-based diet and exercise on functional outcomes among older, overweight long-term cancer survivors: RENEW: A randomized controlled trial. JAMA 301:1883-1891, 2009

36. Wiskemann J, Dreger P, Schwerdtfeger R, et al: Effects of a partly self-administered exercise program before, during, and after allogeneic stem cell transplantation. Blood 117:2604-2613, 2011

37. Demark-Wahnefried W, Morey MC, Sloane R, et al: Reach out to enhance wellness home-based diet-exercise intervention promotes reproducible and sustainable long-term improvements in health behaviors, body weight, and physical functioning in older, overweight/obese cancer survivors. J Clin Oncol 30:2354-2361, 2012

38. Rajotte EJ, Yi JC, Baker KS, et al: Communitybased exercise program effectiveness and safety for cancer survivors. J Cancer Surviv 6:219-228, 2012 


\section{Acknowledgment}

We thank all the patients and families who have generously provided information over the years, the Washington State Department of Health for providing data access, and the Fred Hutchinson Cancer Research Center long-term follow-up team, including Peggy Adams-Myers, Kate Chilson, Kathleen Erne, and Candace Hamilton for their expert assistance in collecting and assembling the data.

\section{Appendix}

\begin{tabular}{|c|c|c|c|c|c|}
\hline \multirow[b]{2}{*}{ Outcome } & \multicolumn{2}{|c|}{ Responders $(n=543)$} & \multicolumn{2}{|c|}{ Nonresponders $(n=290)$} & \multirow[b]{2}{*}{$P$} \\
\hline & No. & $\%$ & No. & $\%$ & \\
\hline Ischemic heart disease & 18 & 3.3 & 8 & 2.8 & .84 \\
\hline Cardiomyopathy & 14 & 2.6 & 6 & 2.1 & .81 \\
\hline Stroke & 4 & 0.7 & 2 & 0.7 & 1.00 \\
\hline Hypertension & 60 & 11.0 & 46 & 15.9 & .05 \\
\hline Dyslipidemia & 34 & 6.3 & 16 & 5.5 & .76 \\
\hline Diabetes & 22 & 4.1 & 16 & 5.5 & .38 \\
\hline Any of above & 101 & 18.6 & 65 & 22.4 & .20 \\
\hline
\end{tabular}

Abbreviation: HCT, hematopoietic cell transplantation

Table A2. Risk of Cardiovascular and Related Outcomes Among HCT Survivors of $\geq 1$ Year Compared With NHANES Participants (referent)

\begin{tabular}{|c|c|c|c|c|c|c|c|c|c|c|c|c|}
\hline \multirow[b]{2}{*}{ Model } & \multicolumn{2}{|c|}{$\begin{array}{l}\text { Ischemic Heart } \\
\text { Disease* }\end{array}$} & \multicolumn{2}{|c|}{ Cardiomyopathy* } & \multicolumn{2}{|c|}{ Stroke* } & \multicolumn{2}{|c|}{ Hypertensiont } & \multicolumn{2}{|c|}{ Dyslipidemia† } & \multicolumn{2}{|c|}{ Diabetest } \\
\hline & OR & $95 \% \mathrm{Cl}$ & OR & $95 \% \mathrm{Cl}$ & OR & $95 \% \mathrm{Cl}$ & OR & $95 \% \mathrm{Cl}$ & OR & $95 \% \mathrm{Cl}$ & OR & $95 \% \mathrm{Cl}$ \\
\hline $\begin{array}{l}\text { Model two (adjustment for lifestyle } \\
\text { factors)§ }\end{array}$ & 0.8 & 0.5 to 1.1 & 1.9 & 1.1 to 3.4 & 2.7 & 1.5 to 4.6 & 1.8 & 1.4 to 2.3 & 2.3 & 1.8 to 3.0 & 2.0 & 1.4 to 2.8 \\
\hline \multicolumn{13}{|l|}{ Model two stratified by type of HCT } \\
\hline \multicolumn{13}{|l|}{ Model two stratified by GVHD status } \\
\hline No chronic GVHD & 0.6 & 0.3 to 1.1 & 2.8 & 1.3 to 6.4 & 1.8 & 0.8 to 4.0 & 2.4 & 1.7 to 3.4 & 2.8 & 2.0 to 4.0 & 2.5 & 1.6 to 4.1 \\
\hline History of chronic GVHD & 0.9 & 0.6 to 1.5 & 2.0 & 0.98 to 4.1 & 3.4 & 1.8 to 6.3 & 2.4 & 1.8 to 3.2 & 3.1 & 2.3 to 4.1 & 2.8 & 1.9 to 4.3 \\
\hline
\end{tabular}

NOTE. Bold font indicates significance.

Abbreviations: BMI, body-mass index; GVHD, graft-versus-host disease; HCT, hematopoietic cell transplantation; NHANES, National Health and Nutrition Examination Survey; OR, odds ratio.

*Adjusted for concurrent medication use for hypertension, dyslipidemia, and/or diabetes.

†Those who reported taking medication and without history of serious cardiovascular disease (ischemic heart disease, cardiomyopathy, stroke).

ҒAdjusted for sex, nonwhite race/ethnicity, current age, and BMI.

§Model one plus additional adjustment for current smoking, daily fruit/vegetable intake, and physical activity time. 
Table A3. AR\% Associated With Risk Factors for Cardiovascular and Related Outcomes Among Exposed HCT and NHANES Participants*

\begin{tabular}{|c|c|c|c|c|c|c|c|c|c|c|c|c|c|c|}
\hline \multirow[b]{3}{*}{ Outcome } & \multicolumn{14}{|c|}{ Risk Factor } \\
\hline & \multicolumn{2}{|c|}{ Obesity } & \multicolumn{2}{|c|}{ Current Smoker } & \multicolumn{2}{|r|}{ Diet } & \multicolumn{2}{|c|}{$\begin{array}{l}\text { Physical } \\
\text { Activityt }\end{array}$} & \multicolumn{2}{|c|}{ Hypertension } & \multicolumn{2}{|c|}{ Dyslipidemia } & \multicolumn{2}{|c|}{ Diabetes } \\
\hline & $\mathrm{HCT}$ & NHANES & $\mathrm{HCT}$ & NHANES & $\mathrm{HCT}$ & NHANES & $\mathrm{HCT}$ & NHANES & $\mathrm{HCT}$ & NHANES & $\mathrm{HCT}$ & NHANES & $\mathrm{HCT}$ & NHANES \\
\hline Ischemic heart disease & $<1$ & 36 & 37 & 63 & 7 & $<1$ & - & - & 53 & 57 & 77 & 23 & 28 & 32 \\
\hline Cardiomyopathy & $<1$ & 50 & 27 & 65 & 24 & 1 & - & - & 51 & 55 & 62 & 46 & 28 & 52 \\
\hline Stroke & 14 & 36 & 31 & 66 & 8 & $<1$ & - & - & 16 & 93 & 46 & $<1$ & 10 & 8 \\
\hline Hypertension $\neq$ & 45 & 68 & $<1$ & $<1$ & $<1$ & 29 & 21 & 26 & & & & & & \\
\hline Dyslipidemiał & 34 & 62 & 6 & $<1$ & 8 & 29 & $<1$ & $<1$ & & & & & & \\
\hline Diabetesł & 48 & 82 & 25 & 16 & 17 & 49 & 17 & 43 & & & & & & \\
\hline
\end{tabular}

Abbreviations: AR\%, attributable risk percent; HCT, hematopoietic cell transplantation; NHANES, National Health and Nutrition Examination Survey.

*Models adjusted for sex, nonwhite race/ethnicity, current age, and risk factors shown.

†AR\% for physical activity in relation to ischemic heart disease, cardiomyopathy, and stroke not shown, given that those outcomes typically lead to reduced capacity for physical activity.

$\ddagger$ Those who reported taking medication and without history of serious cardiovascular disease (ischemic heart disease, cardiomyopathy, stroke). 